\title{
SIMILARITIES BETWEEN MOTION PARALLAX AND STEREOPSIS IN HUMAN DEPTH PERCEPTION*
}

\author{
Brian Rogers and Maureen Graham \\ Psychological Laboratory, University of St Andrews, St Andrews, Scotland
}

(Receired 15 Mal 1981)

\begin{abstract}
Random dot techniques were used to investigate the human visual system s sensitivity to sinusoidal depth modulations specified by motion parallax information. Thresholds for perceiving depth were found to be smallest when the spatial frequency of the depth corrugations was between 0.2 and $0.5 \mathrm{c} / \mathrm{deg}$ visual angle. These data were compared with the equivalent thresholds for perceiving depth corrugations specified by binocular disparity using similar apparatus and psychophysical procedures. The similarity between the sensitivity functions is suggestive of a closer relationship between the two systems than has previously been thought.
\end{abstract}

\section{INTRODUCTION}

Over the past twenty years, many valuable insights have been gained into the mechanisms and processes of stereopsis through the use of random dot stereograms (Julesz, 1960, 1971). The unique characteristic of this method of portraying stereoscopic information lies in the fact that there are no monocular cues as to the form or shape of the 3-D surface. Several years ago, the authors set out to investigate whether similar random dot techniques could be used to study depth from relative movement or motion parallax, in the human visual system (Rogers and Graham, 1979). Essentially their technique involved the use of a single random dot pattern. viewed monocularly, which is transformed (thereby generating relative motion on the retina) with every movement of the observer's head. When the observer's head is stationary, there is no information in the random dot array that could allow the observer to judge or infer the shape of the three dimensional surface specified. However, as soon as the observer's head is moved, relative movement is generated between the elements making up the dot pattern which would exactly match the motion parallax produced by a real three dimensional surface Under these conditions, subjects typically reported that the random dot array appeared as a solid and stationary 3-D surface which was "attached" to the oscilloscope screen. The relative movement between the rows of dots in the display was not perceived. On the basis of these demonstrations, Rogers and Graham (op cit) concluded that motion parallax can provide an accurate, powerful and unambiguous source of information about the structure of three dimensional objects and surfaces. Other authors, notably Braunstein $(1966,1968,1976)$ have come to similar conclusions about the efficacy of relative motion

* A preliminary report of these findings was presented at the 1980 ARVO conference in Orlando. Florida. when the observer is stationary and the simulated 3-D object moves across his line of sight. More recently, Ullman (1979) has shown mathematically that there is sufficient information in a sequence of only three discrete views of four points to uniquely specify the three dimensional structure of any rigidly moving surface.

In their original paper, Rogers and Graham reported that the amount of depth perceived in a simulated three dimensional surface varied directly with the amount of relative motion in the display, as one would expect from theoretical considerations. This suggests that motion parallax can function as at good quantitative as well as qualitative indicator of the shape of three dimensional surfaces. But what are the limits on our ability to use parallax information and how do these compare with our stereoscopic abilities? The experiments described in this paper were designed to answer these two questions. First. we wanted to determine the visual system's absolute sensitivity to sinusoidal depth corrugations as a function of the spatial frequency of the corrugations, when the depth information was specified only by relative motion. Second. we wanted to compare the sensitivity function for depth from motion parallax with the equivalent function for depth from binocular disparity.

The rationale behind making such a comparison is based on two factors. First, the subjective appearance of the depth effects derived from parallax information is very similar to the impression of depth obtained from a random dot stereogram (Rogers and Graham. op cit). Second, and more important, there is a close formal similarity between the two systems which is reflected in the nature of the tasks which are carried out in the two cases. For stereopsis, the task is to detect the small differences or disparities between the position of any corresponding object stimulating the two retinae simultaneously, whilst for parallax, it is to detect the difference in position or relative motion of any corresponding object stimulating the single retina 
successively over time. Expressed another way, if we consider the two retinal images at the beginning and end of a head movement through the distance separ. ating the eyes, these are identical to the two images simultaneously stimulating the retinae in a stereoscopic view of the same scene.

In both the parallax and stereoscopic experiments reported here, we measured sensitivity as a function of the spatial frequency of the corrugations in depth. The use of these depth gratings is therefore analogous to the use of spatial luminance gratings to measure contrast sensitivity in the spatial frequency domain (Campbell and Robson, 1968). In the case of luminance gratings, thresholds may be expressed in terms of the smallest peak to peak contrast necessary for the detection or recognition of spatial structure. In the depth domain. thresholds are based on the smallest peak to peak modulation in depth that can be perceived, as a function of the number of corrugations or the spatial frequency of the three dimensional surface. Tyler (1974) first reported that the sensitivity to random dot disparity gratings decreases substantially when the spatial frequency of the corrugations is greater than $1 \mathrm{c} / \mathrm{deg}$ visual angle. In some later work using line stereograms rather than random dot patterns, Tyler (1975) showed that the sensitivity function for line stimuli which are sinusoidally modulated in depth, exhibits both a high and low frequency fall-off in sensitivity. In this respect, the stereoscopic system behaves in a similar way to the contrast mechanisms described by Campbell and Robson (op cit), except that the peak or optimum spatial frequency for disparity gratings is considerably lower than that found in the luminance domain. More recently, Schumer and Ganz (1979) have extended Tyler's earlier work to include a wider range of spatial frequencies of depth corrugations, as well as providing evidence for the existence of independent channels in the processing of stereoscopic information.

The aim of the experiments reported here was to derive the sensitivity functions for depth from both motion parallax and stereopsis using similar displays and psychophysical procedures in order to make a legitimate comparison between the characteristics of the two systems.

\section{METHODS}

\section{Motion parallax display}

The particular technique used to generate depth from motion parallax information has been described extensively elsewhere (Rogers and Graham, op cit). The aim was to simulate the patterns of relative motion that would be produced by a real three dimensional surface during active movements of the observer's head. To do this, a single, two dimensional random dot pattern, viewed monocularly to avoid conflicting stereo cues, was systematically distorted or transformed with each movement of the observer's head. The random dot pattern was displayed on a
Hewlett Packard 1304A large screen display uscilloscope located $57 \mathrm{~cm}$ from the subject's eye, so that the screen subtended 25 by 20 (horizontal by verticali of visual angle (Fig. 1). The random dot pattern was generated on a Matrox ALT 256 graphics board mounted in a Cromemco System 3 microcompuler. The computer was used to load a particular array of 1 's and 0 's into the display momory as well as Guntrolling the sequence of events in the experiment and recording the subject's responses. The pattern was displayed on the oscilloscope screen using a raster fun where the $\mathrm{X}$ and $\mathrm{Y}$ deflection signals were triggered by line and frame sync pulses originating in the Matrox board. The display was refreshed at a rate of $50 \mathrm{~Hz}$.

Throughout the present experiments, static (unchanging) dot patterns were used to provide the parallax information, although we have discovered in previous work that depth from parallax information can still be perceived when the display is dynamic and each dot has an average life of less than half a second. As yet we do not know the precise point where depth breaks down in these dynamic parallax displays, but it is clear that the elements must survive for a sufficient length of time in order to generate the necessary motion information. Hence parallax depth could not be seen in a T.V. "snowstorm" picture where each point only persists for one frame.

In order to simulate the parallax motion that would be produced by a real three dimensional surface, the random dot array was systematically distorted with each movement of the observer's head. This was accomplished by modulating the amplitude of an additional waveform fed to the $X$ input of the display oscilloscope. For example. to simulate a sinusoidally corrugated surface (Fig. 2), the distortion signal was a sine wave whose amplitude was modulated by a voltage derived from a potentiometer attached to the chinrest. The parallax motion was therefore continuous and exactly in step with each movement of the subject's head. Thus as the subject moved his head from left to right, several horizontal bands of dots (representing the troughs of the corrugations) would move in the same direction across the screen, whilst those representing the peaks of the corrugations would move in the opposite direction. Between the peaks and troughs, the amount and direction of rela" tive motion was interpolated correctly according to the shape of the simulated surface. Note that there was only relative motion between the dots whilst the subject's head was moving - at all other times the dot pattern was static and yielded no information as to the shape of the simulated three dimensional surface. The pattern was always slightly larger than the dimensions of the screen so that the edges of the pattern (which contain information about the shape of the simulated surface) were never visible. The parallax or distortion signal was generated by a Wavetek 175 arbitrary waveform generator, synchronised 10 the frame rate of the display. By varying the waveform of 


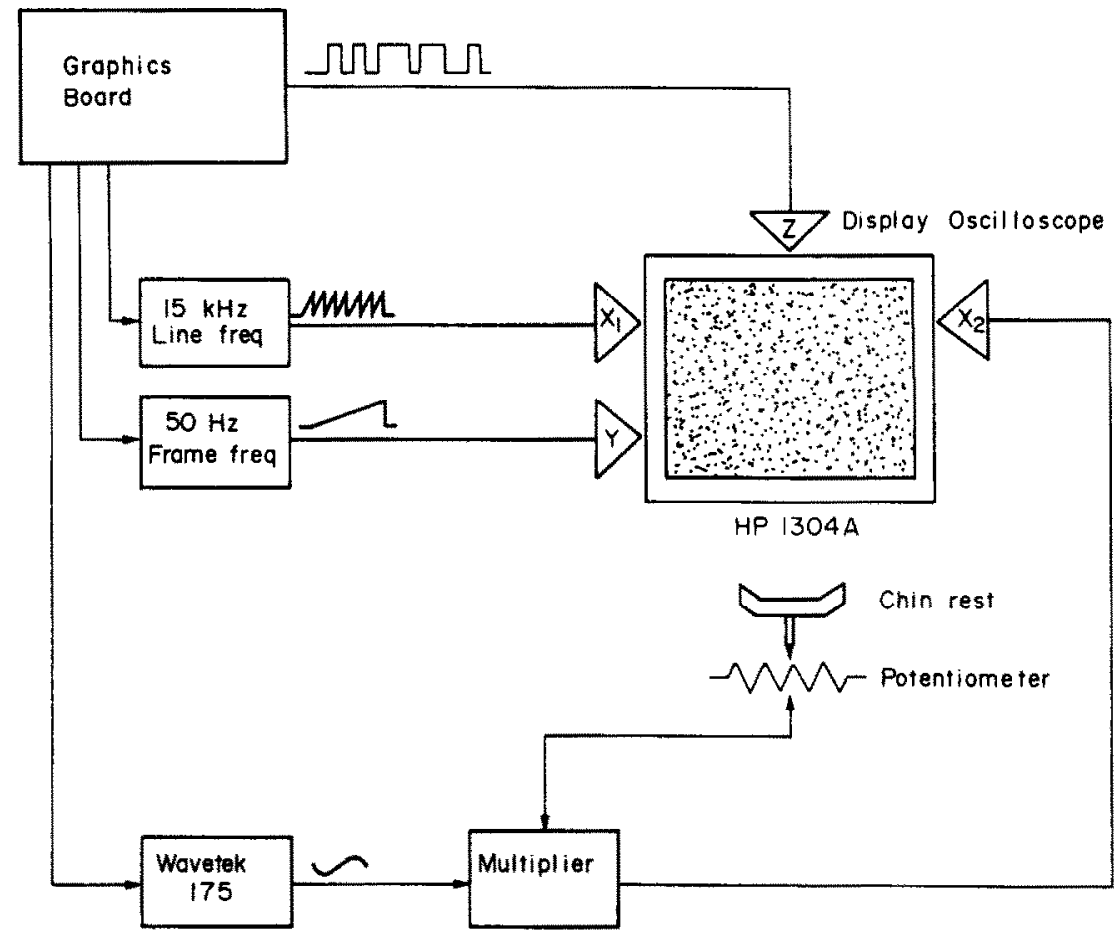

Fig. 1. A static random dot pattern was displayed on the oscilloscope screen using a raster technique. The Matrox ALT 256 graphics board provided the $Z$ (intensity) signal as well as the sync pulses for the line (X1) and frame (Y) ramp generators. The entire pattern could be "distorted" by an additional waveform fed to the $\mathrm{X} 2$ input, whose amplitude was controlled by the position of the subject's head on the chin rest.

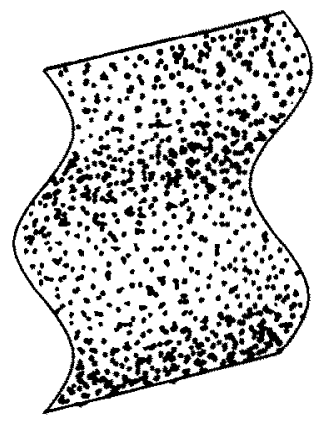

(a)
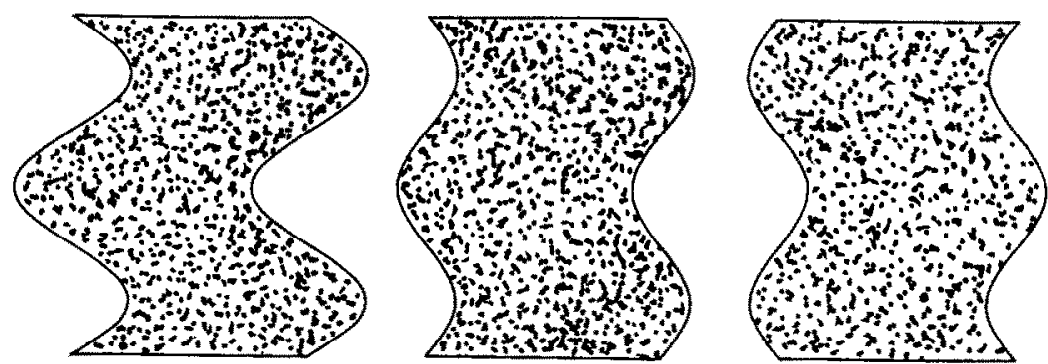

(b)

Fig. 2. A perspective impression (a) of a sinusoidally corrugated surface which was used in the threshold measurements for both motion parallax and stereoscopic depth. To simulate the relative movement that would be produced by a real 3-D surface. the 2-D random dot pattern was systematically "distorted" in the exaggerated sequence shown underneath as the subject moved his head from left to right (b). The actual displacement was always continuous and exactly in step with the head movement. 
the distortion signal, the shape of the surface represented in the parallax transformation could be altered.

Two other characteristics of the parallax surfaces could also be manipulated. First, the number of cycles or simulated corrugations could be varied according to the temporal frequency of the parallax signal. In the present experiments, between 1 and 32 complete cycles or corrugations in depth were used, corresponding to spatial frequencies of between 0.05 and $1.6 \mathrm{c} / \mathrm{deg}$ of visual angle. Second, the amount of relative depth specified in the transformation could be varied. In a real three dimensional surface, the amount of parallax motion between the peaks and troughs of the surface is directly proportional to the amount of relative depth in that surface. Hence by altering the gain of the amplitude modulation, more or less relative motion could be introduced into the display for the same extent of head movement. In these experiments, the chin rest was always constrained to move laterally through a maximum distance of $15 \mathrm{~cm}$. Subjects viewed the display monocularly and were allowed to move their heads from side to side at their own preferred rate. This was typically about one oscillation every $2 \mathrm{sec}$. Subjects were also allowed to freely scan the dot pattern or fixate on a particular element. The threshold measurements did not appear to be influenced significantly by the pattern of eye movements. or by the precise velocity of head movement.

\section{Stereoscopic display}

Random dot patterns were again used in the stereoscopic display to portray three dimensional corrugated surfaces. In this case, however, the information used to specify the shape of the 3-D surface was provided by the disparities between the random dot patterns presented to the two eyes (Julesz. 1971. Tyler, 1974). The dot patterns were presented on two HP 1304A display oscilloscopes viewed stereoscopically via a pair of mirrors in front of the subject's cyes and at 45 to the line of sight. As in the parallax experiments. the random dot arrays were generated on a $256 \times 256$ matrix with a $50^{\circ} \%$ probability of each element being illuminated. Initially, the random dot patterns seen by the two eyes were identical, so that when fused, subjects perceived a single flat array of random dots lying in the plane of the oscilloscope face. The angular subtense of the patterns and the overall dot density were identical to those used in the motion parallax display.

Three dimensional corrugated surfaces were produced by introducing an additional disparity signal to the $X$ input of one of the display oscilloscopes. For example, to generate a sinusoidally corrugated surface, an additional sine wave signal, synchronised to the frame rate of the raster scan, was added into the $X$ input. This additonal signal caused the rows of dots representing the peaks and troughs of the corrugated surface to be displaced horizontally with respect to the screen and to the other dot pattern. thereby ureating binocular disparities. By altering the waveform of this disparity signal, the shape of the stereoseopic surface could be varied.

As in the parallax experiments. the spatial frequency of the disparity corrugations could be varied by altering the temporal frequency of the disparity signal. Between one and thirty two cycles or currugations could be displayed using this technique, corresponding to spatial frequencies of between 0.05 and $1.6 \mathrm{c} / \mathrm{deg}$ visual angle. The amount of depth in the corrugated surface was specified by the amplitude of the disparity signal which affected the amount of horizontal displacement and thus the disparity between corresponding elements in the two displays.

\section{Procedure}

The preceding discussion has emphasised that. apart from the differences in the technique used to specify the depth in the corrugated surface, all other characteristics of the parallax and stereoscopic displays were identical. Similarly, the psychophysical procedures for obtaining the threshold measurements were identical in both experiments. Each subject was presented with a series of 48 trials in a single session. comprising 8 repetitions at each of 6 different spatial frequencies in a randomised sequence. An ascending method of limits was used, such that the parallax or disparity signal was slowly increased in amplitude until the subject was able to perceive the depth in the random dot surface and correctly report the number of corrugations and their phase. This rather conservative procedure was chosen to ensure that the judgements were made on the basis of perceived depth and not, for example, on the detection of relative motion or displacement. The only difference between the procedure used in the two experiments was that the subject remained stationary and viewed the random dot patterns binocularly in the stereo experiment. whilst for motion parallax, a single random dot pattern was viewed monocularly and the subject moved his head from side to side until depth was perceived on a particular trial. Stereoscopic and motion parallax thresholds were recorded on different days. Three observers including the two authors acted as subjects in both experiments.

\section{RESULTS}

\section{Motion parallax gratings}

On each separate trial, subjects pressed a key to indicate when they could perceive the three dimensional structure of the surface and report on the number of depth corrugations present. The amount of relative motion between the peaks and troughs of the simulated corrugations at that moment was recorded and later averaged with the other thresholds at the same spatial frequency. The mean threshold values for two subjects are presented in Fig. 3 as a function of the spatial frequency of the depth corrugations. The 


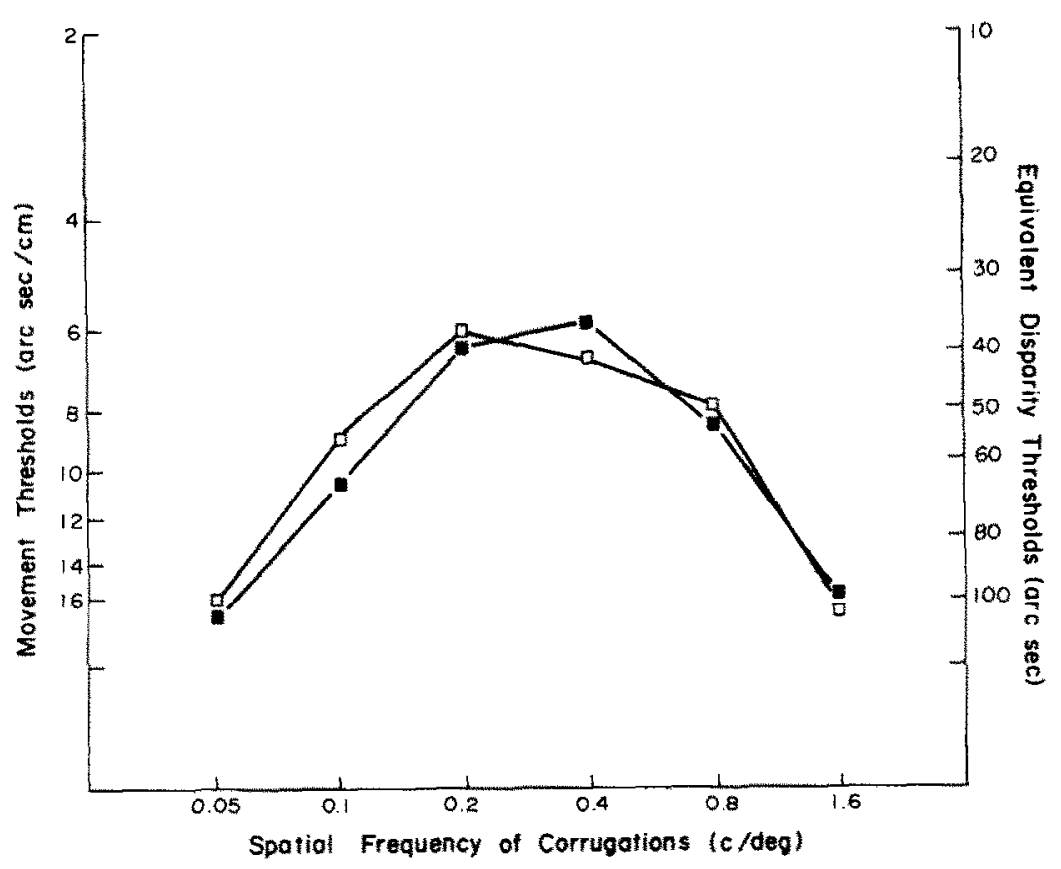

Fig. 3. Threshold measurements for perceiving depth from motion parallax as a function of the spatial frequency of the depth corrugations. Maximum sensitivity occurs when the spatial frequency of the corrugations is about $0.2-0.4 \mathrm{c} / \mathrm{deg}$. Open symbols represent the data of subject BJR and closed symbols $M E G$ in this Figure and Fig. 4

abscissa is calibrated in c/deg of visual angle on a logarithmic scale, whilst the ordinate is expressed in terms of the amount of relative motion between the peaks and troughs of the pattern surface in are seconds for each centimeter of head movement. On the right-hand side of the graph, the ordinate is calibrated alternatively in terms of the equivalent disparity in order to make a direct comparison with the stereo thresholds. If we consider a real three dimensional surface it is possible to calculate both the binocular disparity difference between the peaks and troughs of the corrugations, and the amount of relative motion that would be produced for a given extent of lateral head movement. In the special case of a head movement through the distance separating the eyes, the total relative displacement (in arc sec) between the elements representing the peaks and troughs of the surface is identical to the difference in binocular disparity (in arc sec) between the peaks and troughs of the same surface viewed stereoscopically. Hence the thresholds derived for depth from motion parallax can be transformed directly into the equivalent disparities for a stereoscopic surface.

The first point to note about the data presented in Fig. 3 is that the function relating sensitivity to spatial frequency shows a peak around $0.2-0.5 \mathrm{c} / \mathrm{deg}$ visual angle, with a fall-off in sensitivity at boih lower and higher spatial frequencies. In this respect the functions are comparable with those reported by Campbell and Robson (1968) and others for detecting luminance grating patterns. However, whereas the peak sensitivity for luminance gratings in human observers lies between 3 and $5 \mathrm{c} / \mathrm{deg}$, the sensitivity function for depth gratings specified by motion parallax are shifted downwards by a factor of 10 . In the region of peak sensitivity for these depth gratings, the threshold amount of relative motion between the parts of the display representing the peaks and troughs of the corrugations, is less than 5 arc sec for each $\mathrm{cm}$ of head movement. Expressed another way, a three dimensional surface of that spatial frequency could be detected at $57 \mathrm{~cm}$ if the difference in depth between the peaks and troughs was less than $1 \mathrm{~mm}$.

\section{Stereoscopic gratings}

Thresholds for perceiving depth gratings when the depth was specified by binocular disparities are shown in Fig. 4. The abscissa again indicates the spatial frequency of the corrugations whilst the ordinate is calibrated in arc seconds of dispartity between the peaks and troughs of the surface. The sensitivity function function for stereoscopic gratings shows a peak between 0.2 and $0.5 \mathrm{c} / \mathrm{deg}$, with a fall-off in sensitivity at both lower and higher spatial frequencies. These results confirm and extend those of Tyler (1974) and Schumer and Ganz (1979), both in terms of the region of peak sensitivity and the absolute sensitivity at that point. In our own data, the maximum sensitivity at $0.4 \mathrm{c} / \mathrm{deg}$ is less than $20 \mathrm{arc} \mathrm{sec}$ of peak to peak disparity in the corrugated surface, using somewhat conservative psychophysical procedures. This corresponds with the ability to perceive depth corrugations in a surface $57 \mathrm{~cm}$ away with less than $0.5 \mathrm{~mm}$ of relative depth between the peaks and troughs. Our 


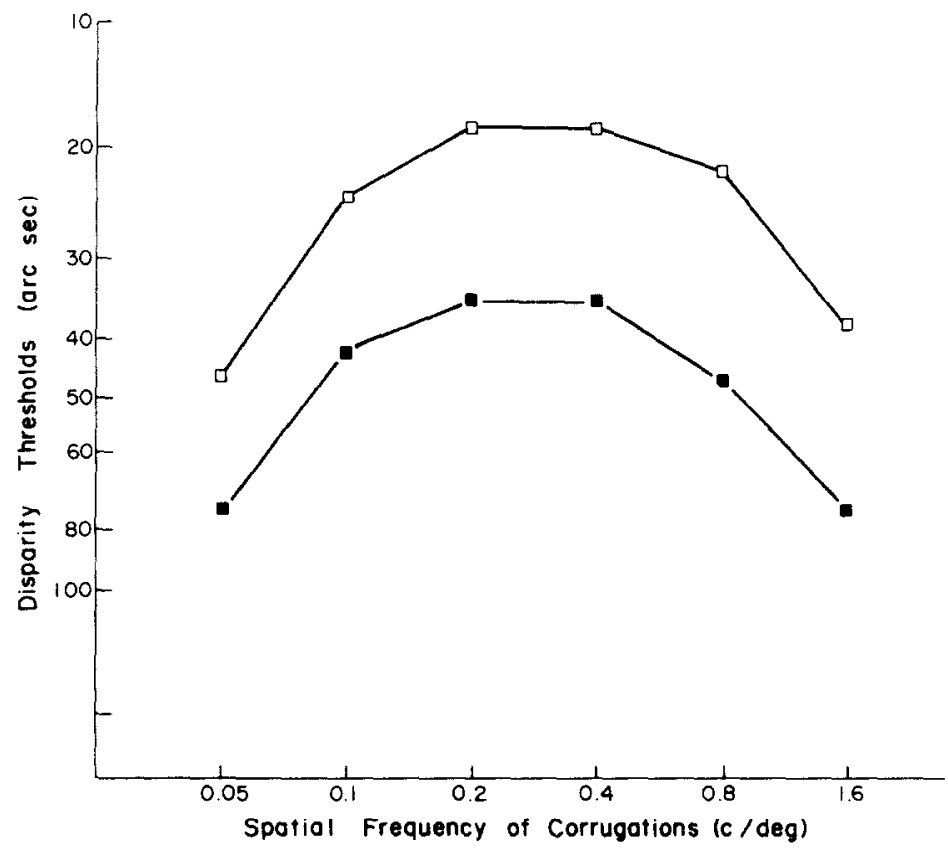

Fig. 4. Threshold measurements for perceiving depth from stereoscopic information as a function of the spatial frequency of the depth corrugations. Like motion parallax, the region of maximum sensitivity also occurs when the spatial frequency of the corrugations is about $0.2-0.4 \mathrm{c} / \mathrm{deg}$.

own observations, and those of Schumer (personal communication) suggest that thresholds can be 2-3 times lower if forced choice procedures are used.

It is immediately apparent from the data presented in Figs 3 and 4 that the shape of the sensitivity functions for depth from motion parallax and stereopsis is very similar. This similarity is made explicit in Fig. 5 where the thresholds for both parallax and stereopsis are plotted on the same graphs for each subject, using the equivalent disparity transformation described earlier. For all three subjects, the shape of the two sensitivity functions are very similar over the entire five octave range of spatial frequencies used in the experiments. Moreover, the absolute sensitivity to depth from motion parallax and stereopsis is at least comparable. For two subjects out of three, sensitivity for perceiving depth from binocular disparities was better by a factor of two compared with that from

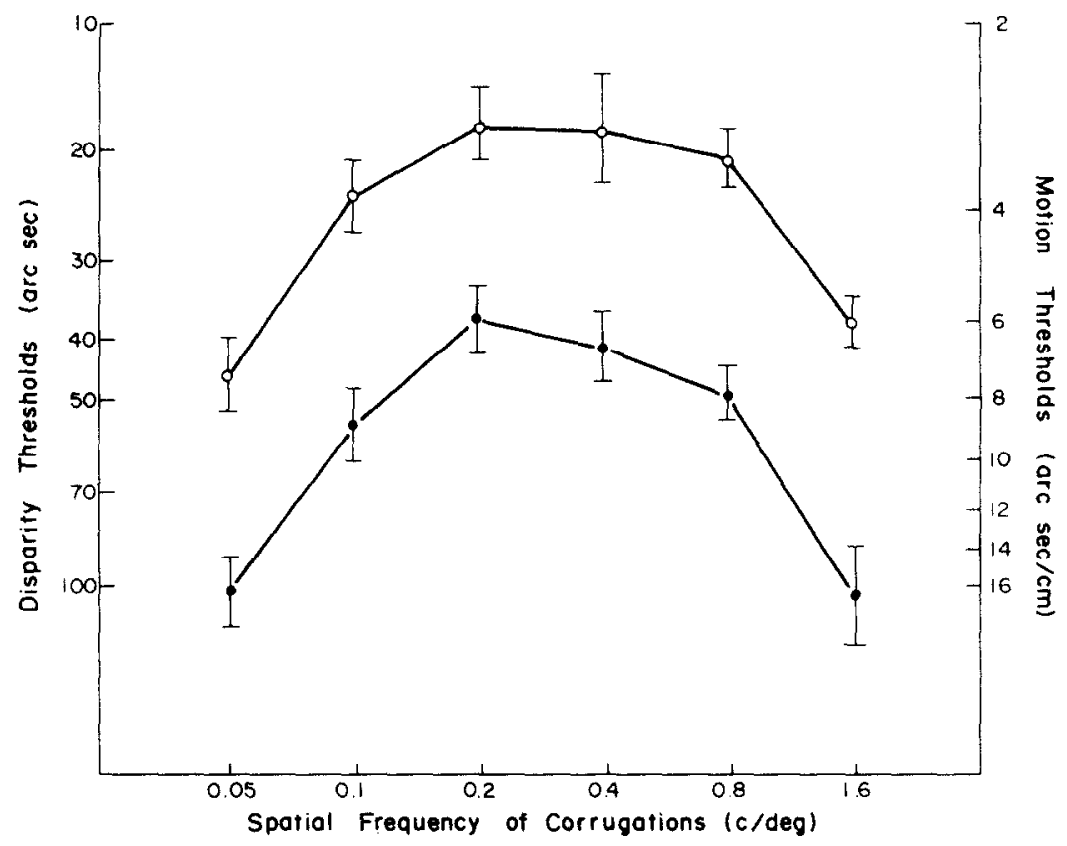

Fig. 5(a). 


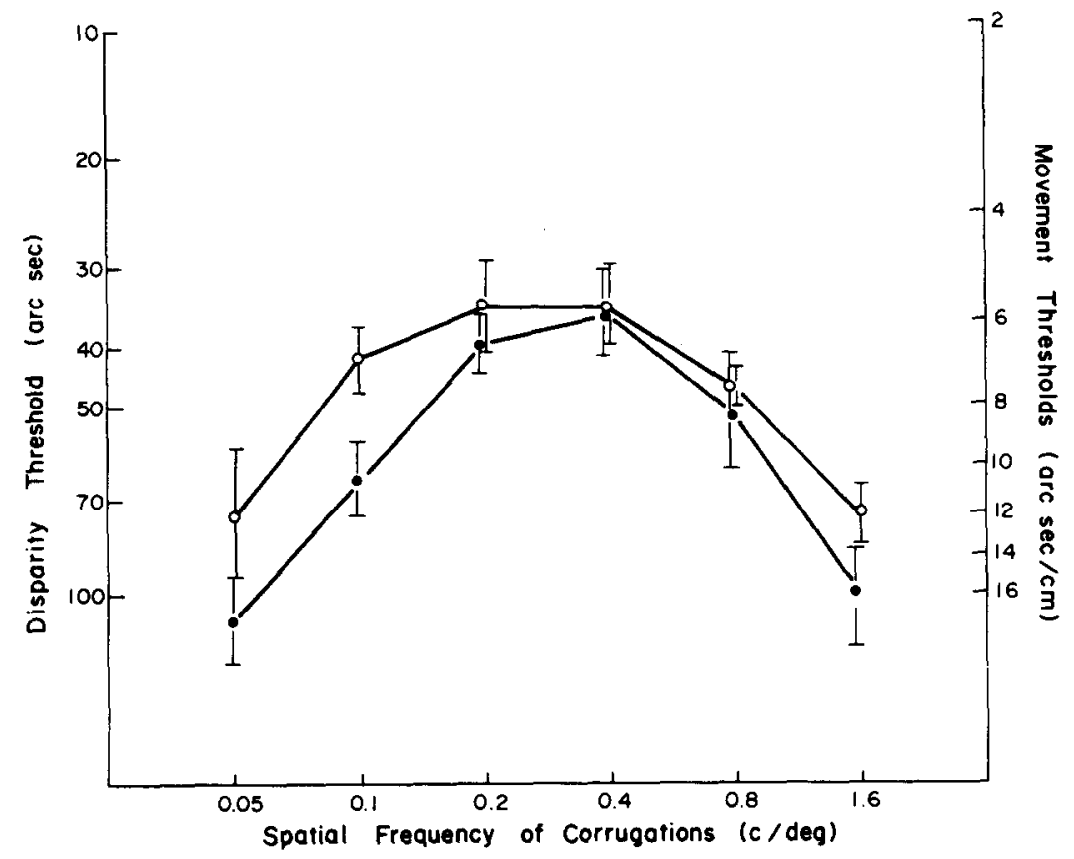

Fig. $5(b)$.

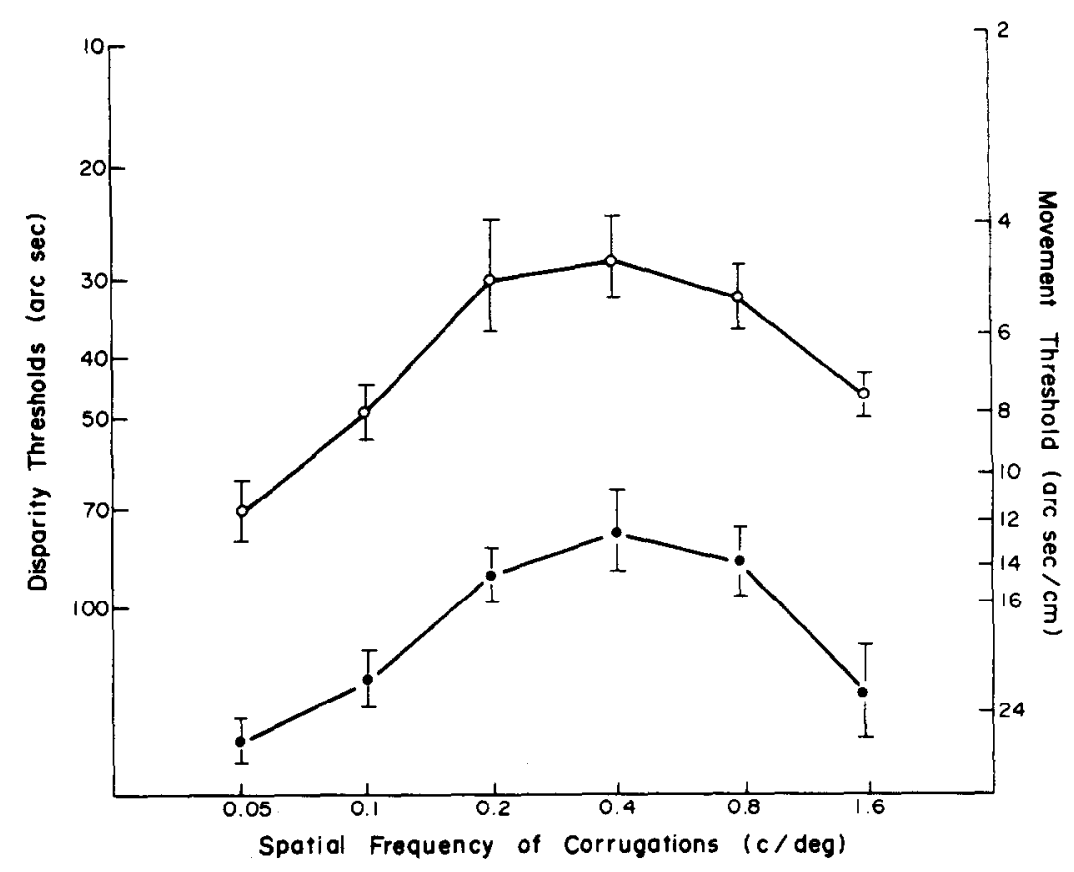

Fig. 5(c).

Fig. 5. A comparison of the thresholds for perceiving depth from motion parallax (closed symbols) and stereopsis (open symbols) for the three subjects. The parallax thresholds are transformed using the procedure described in the text. In all cases, the shape of the sensitivity functions is very similar both in terms of the region of maximum sensitivity and the fall-off in sensitivity at higher and lower spatial frequencies. For two subjects B.J.R. and J.G.Q. (Figs 5a and 5c) thresholds for depth from stereopsis are lower by a factor of two compared with parallax thresholds. The sensitivity functions for the third subject M.E.G. (Fig. Sb) are more similar. 
relative motion, whilst the sensitivity functions for the third subject (MEG) are almost indistinguishable (Rogers et al.,1980).

\section{DISCUSSION}

The experiments described in this paper were designed to investigate two aspects of 3-D perception: first, to determine the absolute sensitivity to depth from motion parallax and second, to compare the visual system's ability to interpret the structure of three dimensional surfaces when the depth information is specified by motion parallax, on the one hand, and binocular disparities on the other. Our threshold data show that the visual system can pick up relative motion between different parts of the display during self-produced movements of the head, when the displacement between the elements of the pattern over 1 apart is less than 5 arc sec for each $\mathrm{cm}$ of head movement. Moreover. the ability to interpret depth from parallax information extends at least over a five octave range of corrugation frequencies from 0.05 to $1.6 \mathrm{c} / \mathrm{deg}$.

The sensitivity curves for perceiving depth from stereoscopic information agree well with the results from previous studies. Tyler (1974) demonstrated the high spatial frequency fall-off in sensitivity above $1 \mathrm{c} / \mathrm{deg}$ using random dot patterns with spatial frequency and amplitude swept disparity corrugations. In another paper Tyler (1975) also measured sensitivity for depth corrugations using line stimuli and found that the region of maximum sensitivity was between 0.3 and $1 \mathrm{c} / \mathrm{deg}$ for his two subjects. Absolute sensitivity in that experiments was between 15 and 30 arc sec of disparity between the peaks and troughs of the corrugations. In more recent work by Schumer and Ganz (1979) thresholds for perceiving depth in dynamic random dot stereograms were found to be as low as $15 \mathrm{arc} \mathrm{sec}$ with brief $(60-100 \mathrm{msec})$ presentations. Again their results are very comparable with our own data despite considerable differences in the stimulus and display characteristics.

In the results from both the parallax and stereo experiments, there is considerable fall-off in sensitivity when the spatial frequency of the corrugations is above $1 \mathrm{c} / \mathrm{deg}$. It could be argued that the poorer sensitivity is due to limitations based on the size of the elements making up the dot pattern. In fact, in our experiments, there were never less than cight rows of dots specifying each complete sinusoidal corrugation. Moreover, from other experiments we have found that doubling the dot density has no appreciable affect on threshold values at these higher spatial frequencies. On the other hand, it is possible that some of the low frequency fall-off in sensitivity might be attributable to the small number of depth corrugations visible in the display. In the case of the $0.05 \mathrm{c} / \mathrm{deg}$ surface, only one complete cycle was present and, as Tyler (1975) has demonstrated with depth corrugations based on line stimuli, sensitivity is underestimated when fewer than two complete corrugations are visible. For this reason, the results obtained at these low spatial frequencies should be interpreted with caution. In the luminance domain, van der Wildt, Keemink and van den Brink (1976) have suggested that the detection of low spatial frequency gratings is better considered in terms of the spatial luminance gradients present in the pattern. A similar analysis of the detectability of depth gradients derived from either stereoscopic or parallax information would be extremely valuable here.

An obvious question that arises. concerns the relationship between the thresholds for perceiving depth from motion reported here, and the thresholds for perceiving differential motion in its own right. Clearly, the visual system must be capable of detecting relative motion at the point at which an observer perceives depth in the parallax situation, but this does not imply that observers would necessarily be able to perceive or detect relative motion with smaller amplitudes. In the case of the motion parallax effects described here, it is apparent that relative motion is not perceived in the parallax surfaces. even when the amount of depth in the surfaces (and the amplitude of relative motion) is considerably above threshold. Instead, observers typically report that the depth corrugations appear to be solid and "fixed" to the oscilloscope screen. and are merely viewed from different angles as the observer moves his head from side to side (Rogers and Graham, 1979). The only exception to this general rule occurs when the spatial frequency of the corrugations is higher than about $2 \mathrm{c}$ deg visual angle. Above this value, relative motion between different rows of dots is frequently perceived before any impression of depth obtains. This implies that the sensitivity function for perceiving relative motion ought to be flatter or show a less severe fall-off in sensitivity at high spatial frequencies compared with the functions reported here for depth from motion parallax. Recent work by Nakayama and Tyler (1981) supports this interpretation.

The sensitivity functions for depth from motion parallax show that, in the region of maximum sensitivity around $0.4 \mathrm{c} / \mathrm{deg}$, the amount of peak to peak displacement between the rows of dots is less than 5 arc sec for each centimeter of head movement. Although this sounds impressive, it is in fact much poorer than the absolute sensitivity to differential motion in random dot patterns reported by Nakayama and Tyler (op cit). However, there are two important differences between the conditions of the two studies. First, the temporal frequency of the relative movement changes in the Nakayama and Tyler study was $2 \mathrm{~Hz}$ compared with a rate of only $0.5 \mathrm{~Hz}(1$ oscillation every $2 \mathrm{sec}$ ) in the present study. Nakayama and Tyler's data indicate that thresholds for perceiving relative motion might be 24 times poorer at the lower temporal frequency. Secondly, the observer's judgements of the depth corrugations in the present experiments were made whilst making side to side 
head movements and with monocular viewing, both of which would tend to increase thresholds significantly.

However, it is important to stress that the patterns of relative motion presented to the subject in the Nakayama and Tyler study were almost identical to those used here. The major difference between the two experiments is that that the observer viewed the display whilst moving his head from side to side in the present experiment rather than remaining stationary as in the Nakayama and Tyler experiment. In the former case, subjects perceived a stationary threedimensional surface with no relative movement between the rows of dots, whilst in the latter case subjects perceived relative motion with presumably little or no impression of depth. Does this imply that the difference in the percept of either depth or relative motion is due to the concurrent movement of the subject's head in the parallax experiments? Our observations suggest that although the movement of the subject's head serves to disambiguate the direction of the depth effects, it is the spatial frequency characteristics of the velocity field together with the temporal frequency of change of that velocity field that determine whether depth or motion is perceived. At relatively high spatial frequencies (above $1-2 \mathrm{c} / \mathrm{deg}$ ) and with a rate of change above $2 \mathrm{~Hz}$, relative motion is perceived with little or no impression of 3-D structure, even when the motion is coupled to the subject's head movement. On the other hand, at lower spatial frequencies and with lower rates of change, depth is frequently perceived rather than relative motion, even when the observer's head is stationary. In this case, a corrugated surface with ambiguous depth is perceived as oscillating about a vertical axis through the centre of the screen (Rogers and Graham, 1979). For shadow cast rotating figures. Caelli $(1979,1981)$ has reported a similar change from 3-D percepts to 2-D oscillations when the temporal frequency is above $1-2 \mathrm{~Hz}$. In addition, the absence of conflicting disparity information with monocular viewing increases the likelihood that depth will be perceived. Thus it remains an open question as to whether thresholds for perceiving relative motion are very much lower than thresholds for perceiving depth from relative motion under identical conditions.

\section{Underlying mechanisms}

What implications do these data have for the mechanisms underlying stereopsis and motion parallax? For example, does the shape of the sensitivity functions for depth from motion or disparity information suggest that there are separate channels each selective to a limited range of spatial frequency depth corrugations, as has been proposed in the luminance domain? Schumer and Ganz (1979) have provided some evidence to support this interpretation in the stereo domain from experiments using subthreshold summation and threshold elevation paradigms. The estimated bandwidth of the depth channels is quite broad, extending over at least three octaves. At present, there is no evidence for spatial frequency tuned channels in motion parallax processing, but it is clear that the spatial extent of the mechanisms underlying both systems is very large. At the optimal spatial frequency of depth corrugations, the maximum differences in disparity or relative motion are separated by over one degree of visual angle on the retina. At the lowest spatial frequencies used, where the depth effects are still very visible, the differences in disparity or relative motion are separated by over $10^{\circ}$. This would suggest that any hypothetical receptive fields used to extract disparity or parallax information must extend over several degrees of visual angle. Nakayama and Tyler (1981) have made a similar suggestion about the size of receptive fields underlying motion detection. The receptive fields for both stereo and parallax might receive excitatory inputs from local detectors (in different retinal regions) responding preferentially to opposite directions of motion or retinal disparity. These units would therefore be optimally sensitive to differential velocities or differential disparities over space, and would respond to any changes in depth or depth gradients in the visual world. Alternatively, the receptive fields might have inhibitory inputs from local detectors in neighbouring regions of the retina responding to the same direction of motion or retinal disparity, which would be similarly optimised to respond to depth changes in the visual world. On the basis of rather different evidence, Schumer and Ganz (1979) have proposed similar "cyclopean receptive fields" for disparity processing.

In conclusion, the experiments described in this paper demonstrate some striking similarities between the characteristics of the motion parallax and stereoscopic mechanisms with respect to the detectability of three dimensional corrugated surfaces. Moreover, some recent experimental work has shown that similar and very powerful aftereffects of depth can be produced from both motion parallax and stereoscopic information (Rogers et al., 1981 ; Graham and Rogers, 1981). Likewise, simultaneous contrast effects have been demonstrated in the two domains suggesting that the spatial interactions of the underlying processes are also comparable (Graham and Rogers, op cit). The idea of an underlying similarity between stereoscopic and motion parallax processes is not new and was perhaps implied by Helmholtz (1925) over a hundred years ago, when he argued that the impression of depth obtained from motion parallax was "just as if he were looking at a good stereoscopic view of it" (p 296).

\section{REFERENCES}

Braunstein M. L. (1966) Sensitivity of the observer to transformations of the visual field. $J$. exp. Psychol. 72, 683-689.

Braunstein M. L. (1968) Motion and texture as sources of slant information. J. exp. Psychol. 78, 247-253.

Braunstein M. L. (1976) Depth Perception through Motion. Academic Press, New York. 
Caelli T. M. 11979) On the perception of some geometric properties of rotating three-dimensional objects. Biol. Crhernet 33, 29-37.

Caelli T. M. (1981) Visual Perception Theory and Practice. Pergamon Press, Oxford.

Campbell F. W. and Robson J. G. (1968) Application of Fourier analysis to the visibility of gratings. $J$. Phisiol. 197, 55166

Graham M. E. and Rogers B. J. (1981) Simultaneous and Successive Contrast effects in the perception of depth from motion parallax and stereoscopic information. Perception. In press.

Helmholtz. H. von (1925) Helmholl 's treatise on Physiologi('al Oprics (Edited by Southall J. P.). Optical Society of America. New York.

Julesz B. (1960) Binocular depth perception of computer generated patterns. Bell Syst Tech. J. 39, 1125-62.

Julesz B. (1971) Foundations of Ciclopean Perception. Chicago Press. Chicago.

Nakayama K. and Tyler C. W. (1981) Psychophysical Isolation of Movement Sensitivity by removal of familiar position cues. Vision Re's. 21, 427-433.
Rogers B. J. and Graham M. E. (1979) Motion Parallax as an independent cue for depth perception. Perception $\mathbf{8}$ $125-134$

Rogers B. J., Graham M. E. and Anstis S. M. (1980) Depth perception with Motion Parallax and Disparity Gratings. Incest. Ophthal. visual Sci., Suppl. p. 106.

Rogers B. J., Graham M. E. and Anstis S. M. (1981) Simultaneous and Successive Contrast Effects in depth from stereopsis and motion parallax. Invest. Ophthal. risual Si.. Suppl. p. 223.

Schumer R. and Ganz L. (1979) Independent stereoscopic channels for different extents of spatial pooling. Vision Res. 19, 1303-14

Tyler C. W. (1974) Depth Perception in Disparity Gratings. Nature 251, 140-142

Tyler C. W. (1975) Spatial organisation of binocular disparity sensitivity. Vision Res. 15, 583-590.

Ullman S. (1979) The Interpretation of Visual Motion. M.I.T. Press, Cambridge. MA.

Wildt G. J. van der, Keemink C. J. and Brink G. van den (1976) Gradient detection and contrast transfer by the human eye. Vision Res. 16, 1047-1053. 